University of Nebraska - Lincoln

DigitalCommons@University of Nebraska - Lincoln

Jody Redepenning Publications

Published Research - Department of Chemistry

2-25-2007

\title{
Quantized magnetoresistance in atomic-size contacts
}

\author{
Andrei Sokolov \\ University of Nebraska-Lincoln, sokolov@unl.edu \\ Chunjuan Zhang \\ University of Nebraska - Lincoln \\ Evgeny Y. Tsymbal \\ University of Nebraska-Lincoln, tsymbal@unl.edu \\ Jody G. Redepenning \\ University of Nebraska-Lincoln, jredepenning1@unl.edu \\ Bernard Doudin \\ Institut de Physique et de Chimie des Matériaux de Strasbourg (IPCMS), Strasbourg, France, \\ bernard.doudin@ipcms.unistra.fr
}

Follow this and additional works at: https://digitalcommons.unl.edu/chemistryredepenning

Part of the Chemistry Commons

Sokolov, Andrei; Zhang, Chunjuan; Tsymbal, Evgeny Y.; Redepenning, Jody G.; and Doudin, Bernard, "Quantized magnetoresistance in atomic-size contacts" (2007). Jody Redepenning Publications. 3. https://digitalcommons.unl.edu/chemistryredepenning/3

This Article is brought to you for free and open access by the Published Research - Department of Chemistry at DigitalCommons@University of Nebraska - Lincoln. It has been accepted for inclusion in Jody Redepenning Publications by an authorized administrator of DigitalCommons@University of Nebraska - Lincoln. 


\title{
Quantized magnetoresistance in atomic-size contacts
}

\section{Andrei Sokolov ${ }^{a, b}$, Chunjuan Zhang ${ }^{b, c}$, Evgeny Y. Tsymbal ${ }^{a, b}$, Jody Redepenning ${ }^{b, c}, \&$ Bernard Doudin ${ }^{d}$}

\author{
a Department of Physics and Astronomy, University of Nebraska-Lincoln, Lincoln, Nebraska 68588, USA \\ ${ }^{b}$ Nebraska Center for Materials and Nanoscience, University of Nebraska-Lincoln, Lincoln, Nebraska 68588, USA \\ c Department of Chemistry, University of Nebraska-Lincoln, Lincoln, Nebraska 68588, USA \\ d Institut de Physique et de Chimie des Matériaux de Strasbourg (IPCMS), UMR 7504 CNRS-ULP, 67034 Strasbourg, France \\ Correspondence: Andrei Sokolov, e-mail: asokol@unlserve.unl.edu; \\ Bernard Doudin, e-mail: bernard.doudin@ipcms.u-strasbg.fr
}

Keywords: Electronic properties and devices, Nanomagnetism, spintronics

\begin{abstract}
When the dimensions of a metallic conductor are reduced so that they become comparable to the de Broglie wavelengths of the conduction electrons, the absence of scattering results in ballistic electron transport ${ }^{1}$ and the conductance becomes quantized ${ }^{2}$,

3,4 . In ferromagnetic metals, the spin angular momentum of the electrons results in spin-dependent conductance quantization ${ }^{5,6,}$ 7 and various unusual magnetoresistive phenomena ${ }^{8,9,10,11,12}$. Theorists have predicted a related phenomenon known as ballistic anisotropic magnetoresistance (BAMR) ${ }^{13}$. Here we report the first experimental evidence for BAMR by observing a stepwise variation in the ballistic conductance of cobalt nanocontacts as the direction of an applied magnetic field is varied. Our results show that BAMR can be positive and negative, and exhibits symmetric and asymmetric angular dependences, consistent with theoretical predictions.
\end{abstract}

New developments in spin electronics ${ }^{14,15}$ aim at investigating nanoscale systems for which electron transport properties become significantly different from bulk diffusive models. Tremendous scientific activity in this field has resulted in the design of new electronics architecture using magnetic elements ${ }^{16}$, the electric-field control of spin transport ${ }^{17}$ and a debate regarding the nature of magnetoresistive phenomena in atomic-scale metallic structures ${ }^{18}$. Although the control of composition and magnetism at the atomic level is extremely challenging, a systematic trend in experimental results reveals that nanocontact resistance depends on the relative directions of the saturation magnetization and the electric current ${ }^{19,20,21,22}$. This anisotropy in the resistance is reminiscent of the anisotropic magnetoresistance (AMR) effect that has been known for bulk ferromagnets since the middle of the nineteenth century ${ }^{23}$. Maximum resistivity is found when the magnetization direction is parallel to the electric current and at intermediate angles $\Theta$, a smooth $\cos ^{2} \Theta$ variation is observed. The origin of AMR stems from the anisotropy of scattering produced by the spin-orbit interaction, with stronger scattering of electrons travelling parallel to magnetization, resulting in larger parallel resistivity $^{24}$. The amplitude of the difference is small, and does not exceed $1-2 \%$ for bulk elemental transition metals.

This behaviour changes dramatically in the ballistic transport regime, because there is no electron scattering contributing to the conductance. For a perfect ballistic conductor formed of a wire with a smoothly changing width, the conductance is given by the Landauer formula, $G=N e^{2} / h$ (ref. 25), where $e^{2} / h=1 / 25,813 \Omega^{-1}$ is the conductance quantum per electron spin, and $N$ is the number of open conducting channels, that is, the number of bands crossing the Fermi energy. The latter quantity is influenced by the spin-orbit interaction, which is known to be much stronger in open and constrained geometries than in bulk materials. The ef- fect is anisotropic because the orbital angular momentum is coupled to the spin angular momentum, causing the projection of the former to differ depending on the magnetization direction. By changing the magnetization direction, one can, therefore, change the number of bands at the Fermi energy and thereby modify the ballistic conductance. This phenomenon is called ballistic anisotropic magnetoresistance (BAMR; ref. 13). The angular dependence of BAMR is predicted to be very different compared with AMR. Because the ballistic conductance is an integer multiple of $e^{2} / h$, the BAMR is a step function of the magnetization angle.

To investigate this new phenomenon, we performed in situ measurements of the magnetoresistive properties of electrodeposited nanocontacts bonded to a Si substrate (Fig. 1a,b). This geometry provides excellent mechanical stability and minimizes the influence of magnetostriction and magnetostatic effects on conductance ${ }^{26}$. Conductivity was monitored continuously while opening and closing a contact (Fig. 1c; see also Supplementary Information, Fig. S1). During these events, the conductance plateaux at multiples of $e^{2} / h$ provide a clear signature of the occurrence of ballistic transport. Conductance plateaux lasting up to hundreds of seconds are realized by adequate tuning of deposition (dissolution) at electrochemical reduction (oxidation) potentials of Co. Interestingly, many Co samples show rather stable conductance at 5,6 and $7 e^{2} / h$, consistent with the theoretical predictions for ballistic transport in atomic-size Co wires ${ }^{27}$. Angular-dependence experiments were performed on a contact that did not change during the rotation period. When the conductance corresponding to a ballistic plateau became steady, we initiated the rotation of the sample along the axis in the plane of the substrate perpendicular to the current direction (Fig. 2a) in an external magnetic field of $1 \mathrm{~T}$, sufficient to saturate the magnetization. The striking feature in Fig. $2 \mathrm{~b}$ is the appearance of a stepwise periodic variation of con- 

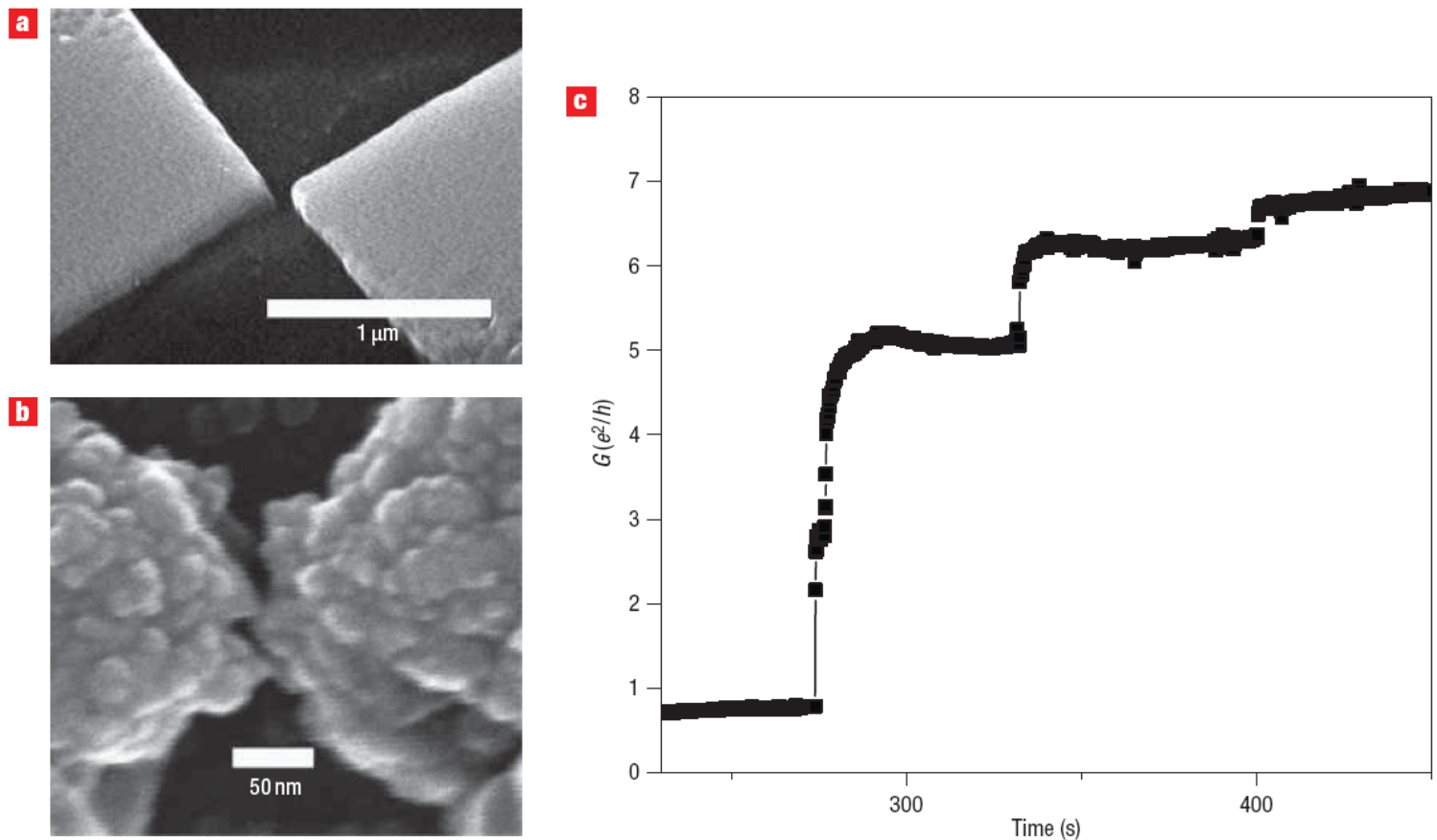

Figure 1: Ballistic transport measurements for Co nanocontacts. a, Micro fabricated arrow-shaped Au electrodes bonded to a Si substrate, with a 100-nm gap patterned prior to Co electroplating. b. High-magnification image of the growth morphology showing the achieved formation of a Co contact area between the electrodes. c, Quantized conductance $G$ during closure of a contact. Conductance plateaux at multiples of $e^{2} / h$ are evident.
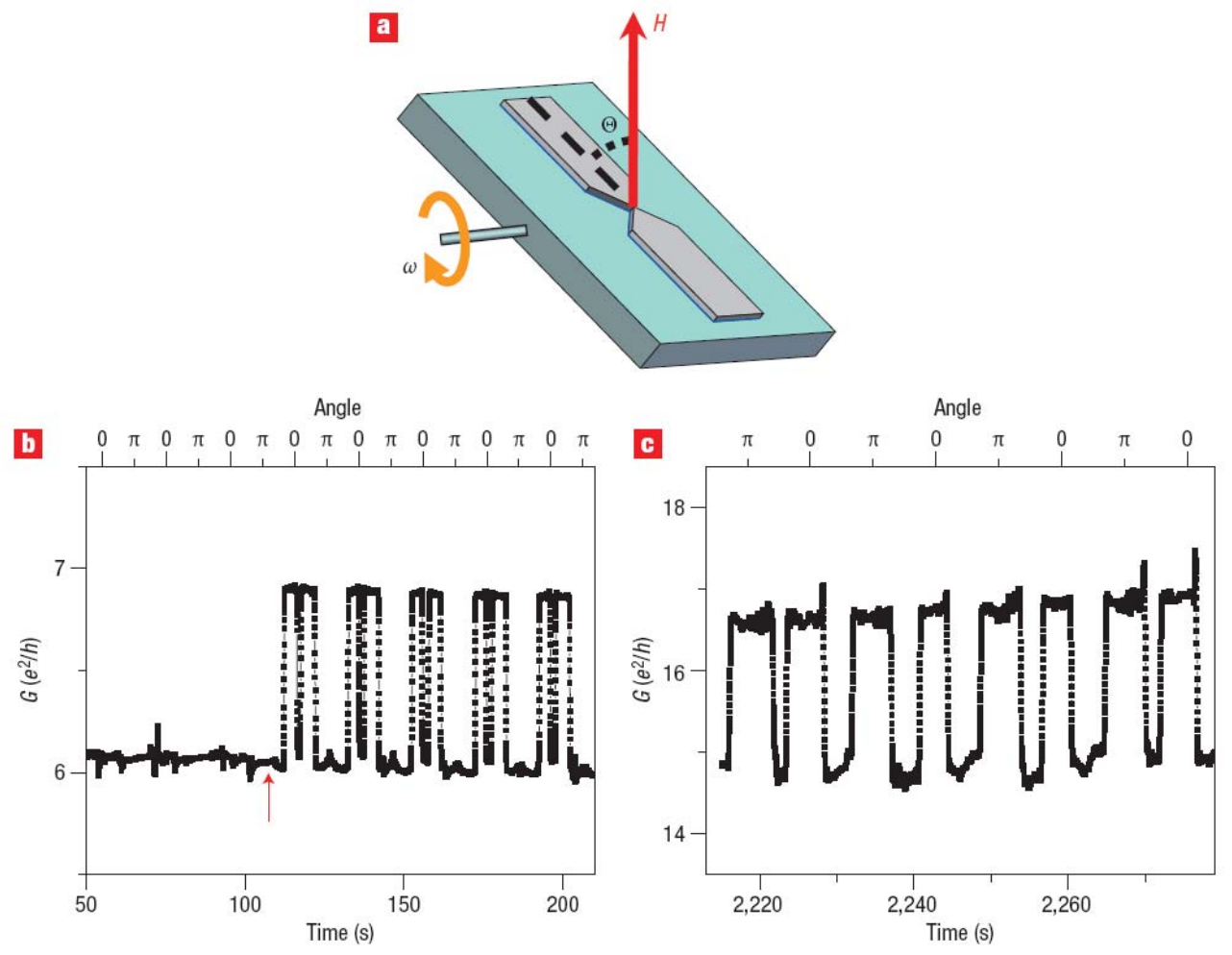

Figure 2: Time-dependent quantum conductance. a, Samples are rotated with angular frequency $\omega=2 \pi / T(T=20 \mathrm{~s})$ in the saturation magnetic field, $\mathrm{H}$, along the axis perpendicular to the electrodes. The angle $\Theta$ is measured with respect to the plane of the electrodes and oscillates between 0 and $\pi$. The conductance measurements are performed during the time interval corresponding to a stable conductance plateau. $\mathbf{b}$, The magnetic field is turned on at an instant indicated by the arrow, after which conductance variations of about one conductance quantum are observed. c, Variations of about two quanta were also observed for a sample with a larger cross-section. 

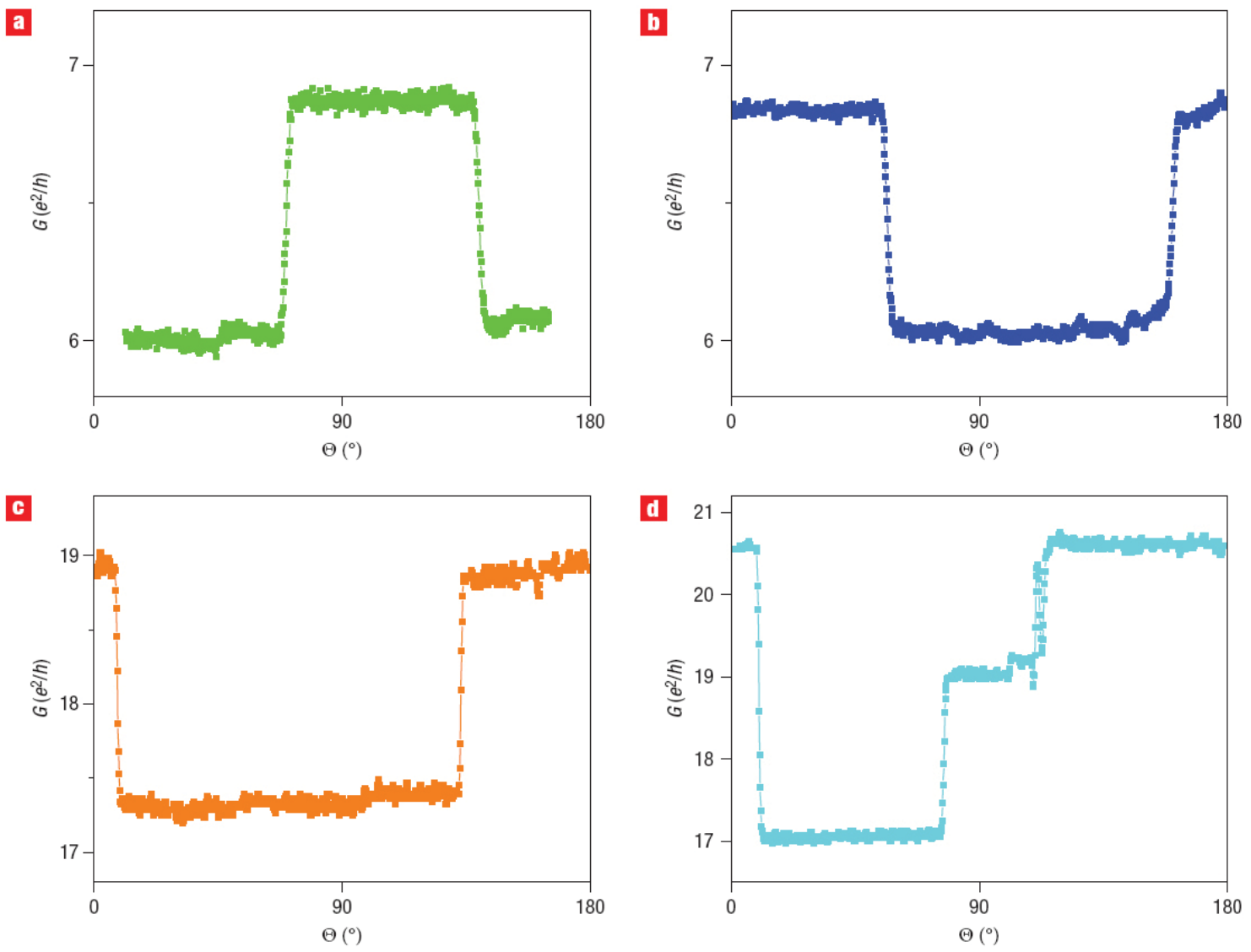

Figure 3: Angular dependence of ballistic conductance. a-d, The angle $\Theta$ between the magnetic field and the sample plane changes from $0^{\circ}$ to $180^{\circ}$. Results for four different samples exhibiting different sign and magnitude of BAMR.

ductance, changing from $6 e^{2} / h$ to $7 e^{2} / h$, that is, by about a spin conductance quantum. Figure $2 \mathrm{c}$ shows another representative conductance versus time curve for a sample with a larger crosssection, exhibiting conductance of about $15 e^{2} / h$, varying by about $2 e^{2} / h$ when rotated under a $1 \mathrm{~T}$ magnetic field.

Figure 3 shows a number of representative curves demonstrating the variety of behaviour we observed in our samples. Figure 3 a shows the angular dependence of conductance for a sample, exhibiting an $e^{2} / h$ step at $\Theta \approx 70^{\circ}$ and a $-e^{2} / h$ step at $\Theta \approx 140^{\circ}$. The angle at which the steps occur varies slightly for different revolutions; however, the shape of the curve is reproducible. For this sample the conductance is higher when the magnetic field is perpendicular to the electrode plane (that is, for $\Theta=90^{\circ}$ ). However, for the majority of samples, a higher conductance is exhibited for a magnetic field parallel to the plane of the electrodes (that is, for $\Theta=0$ ). Two examples are given in Fig. 3b,c in which the conductance appears to be smaller for $\Theta=90^{\circ}$ than for $\Theta=0$. Figure $3 \mathrm{~d}$ displays a more complex behaviour of the conductance as a function of the magnetization angle. Here we observe several steps of magnitude of either $\sim 2$ or 4 conductance quanta within a half revolution. These observed behaviours are thus remarkably different from AMR in amplitude and angular variation. The conductance changes abruptly by a value of nearly 1 or 2 quanta in most occurrences, although in some cases, like the one in Fig. 3d, we observe steps close to $4 e^{2} / h$.

The features observed in the angular variation of conductance can be described using a simple model for the electronic struc- ture of a monoatomic wire (see Methods). Within this model a multiplet of five $d$ orbitals is affected by the crystal field of axial symmetry and spin-orbit coupling. This gives rise to the five electronic bands shown in Fig. $4 \mathrm{a}-\mathrm{c}$ for three different angles $\Theta$ of the magnetization with respect to the wire axis. It is seen that the degeneracy in the band energy occurring at $\Theta=90^{\circ}$ (Fig. 4c) is lifted by spin-orbit coupling at other angles (Fig. 4a,b). This changes the number of bands crossing the Fermi energy $\left(E_{\mathrm{F}}\right)$ with $\Theta$ and consequently the number of conduction channels, thereby producing the BAMR effect. To illustrate different possible scenarios, we assume that the Fermi level can be positioned differently with respect to the bands, as is shown in Fig. $4 a-c$ by the coloured horizontal bars. This may reflect features occurring in real nanocontacts at $E_{\mathrm{F}}$ due to a much more complex electronic structure.

For $E_{\mathrm{F}}$ indicated by the green bar in Fig. $4 \mathrm{a}-\mathrm{c}$, we see that increased splitting of the $E_{2}$ doublet due to the spin-orbit coupling when the angle $\Theta$ changes from $90^{\circ}$ to $0^{\circ}$ leads to a decrease in the number of bands crossing $E_{\mathrm{F}}$ by one. The respective angular variation of the conductance is shown in Fig. 4d. In this case the conductance is higher when the magnetic field is perpendicular to the wire axis. The behaviour is opposite to this for $E_{\mathrm{F}}$ denoted by the blue bar. In this case the number of bands crossing $E_{\mathrm{F}}$ increases by one when $\Theta$ changes from $90^{\circ}$ to $0^{\circ}$, raising the conductance when the magnetic field is parallel to the wire axis. The corresponding angular variation of the conductance is shown in Fig. 4e. 

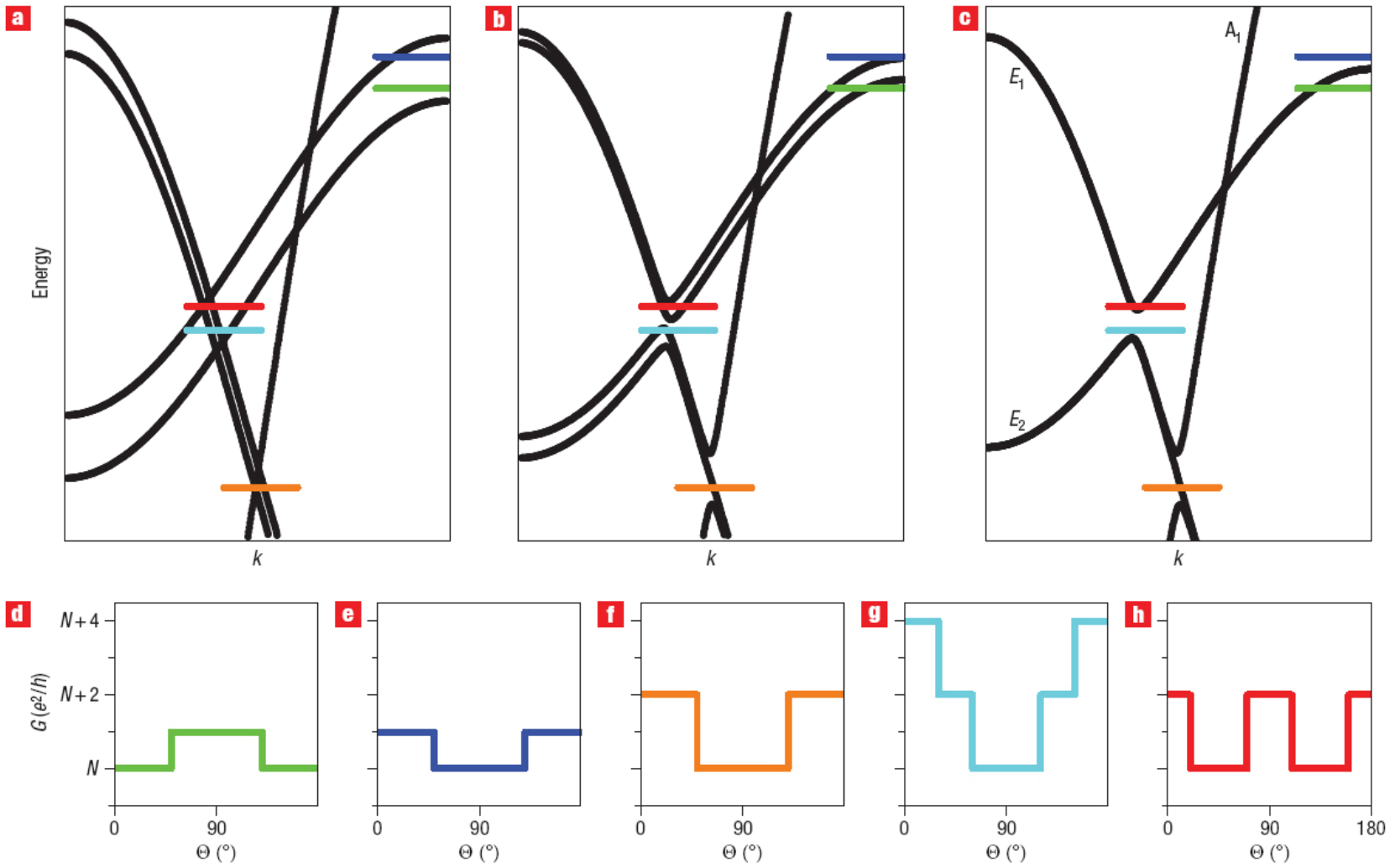

Figure 4: Results of a tight-binding model. a-c, The band structure (band energy $E$ versus wave vector $k$ ) of a monoatomic wire for $\Theta=0^{\circ}$ (a), $\Theta=70^{\circ}$ (b) and $\Theta=90^{\circ}$ (c), where $\Theta$ is the angle between the saturation magnetic field and the wire axis. The degeneracy in the band energy occurring at $\Theta=90^{\circ}$ (c) is lifted by the spin-orbit coupling at other angles (a, b). Colour bars indicate the assumed locations of the Fermi level with respect to the bands. $\mathbf{d}-\mathbf{h}$, Variation of conductance with $\Theta$, shown by curves having the same colour as the corresponding Fermi levels in panels a-c. The symmetry of bands is indicated in panel c (see details in Methods).

A more diverse behaviour is possible if the Fermi level lies at band crossings that are sensitive to the spin-orbit interaction. At the energy marked by the orange bar there are three band crossings for $\Theta=0$ (Fig. 4a). The spin-orbit coupling mixes the $A_{1}$ and $\mathrm{E}_{1}$ bands at $\Theta \neq 0$, creating a pseudogap that removes two crossings (Fig. 4b,c). This results in a conductance step of $2 e^{2} / h$, as shown in Fig. 4f. In the energy region where the two doublets intercept, the angular dependence is even more complex. For $E_{\mathrm{F}}$ denoted by the cyan bar there are two conductance jumps of $2 e^{2} /$ $h$ when $\Theta$ changes from $90^{\circ}$ to $0^{\circ}$ (Fig. $4 \mathrm{~g}$ ). This is also the case for the $E_{\mathrm{F}}$ denoted by the red bar, but in this case the steps are opposite, causing the conductance to be the same at $\Theta=0^{\circ}$ and $\Theta=90^{\circ}$ (Fig. 4h).

This simple model provides the key ingredients necessary to describe the main experimental features. The colour comparison of the curves shown in Fig. 3a-d and Fig. 4d-g illustrates the correspondence between theory and experiment. The asymmetry of the experimental curves not present in the model can be explained by the absence of the full axial symmetry for real nanocontacts. The angle at which the conductance exhibits a step is sensitive to thermal fluctuations within the contact. Thermal fluctuations may populate (depopulate) electronic states near the Fermi level, producing a conductance step at the angle that deviates from that at zero temperature. The angle variation $\Delta \Theta$ can be estimated as $\Delta \Theta /(\pi / 2) \approx k_{\mathrm{B}} T / E_{\mathrm{SO}}$, where the thermal energy at room temperature is $k_{\mathrm{B}} T \approx 0.025 \mathrm{eV}$ and the spin-orbit coupling energy is $E_{\mathrm{SO}} \approx 0.1-0.2 \mathrm{eV}$ (ref. 13). This leads to $\Delta \Theta$ being of the order of a few tens of degrees. The fluctuations of the angle at which the conductance step occurs are seen in Fig. 2b (see also Supplementary Information, Fig. S3).

Our findings unambiguously demonstrate a new magnetoresistance phenomenon specific to quantum ballistic transport. Measurements performed on magnetically saturated atomic-size contacts indicate that a small change in the magnetization direction can cause a large change in conductance, of amplitude and of sign depending on the local atomic configuration. This phenomenon is fundamentally different from known magnetoresistive effects related to the relative reorientation of magnetic moments within the sample.

\section{Experiment}

\section{Methods}

Planar electrodes, of 1-2.5 $\mu \mathrm{m}$ width, and separated by a gap of about $100 \mathrm{~nm}$, were patterned from a Si/SiO${ }_{2} / \mathrm{Ta}_{2} \mathrm{O}_{5}(10 \mathrm{~nm}) / \mathrm{Ni}(100 \mathrm{~nm})$ or $\mathrm{Si} /$ $\mathrm{SiO}_{2} / \mathrm{Ti}(10 \mathrm{~nm}) / \mathrm{Au}(100 \mathrm{~nm})$ wafers using optical lithography and focused ion beam (FIB) milling in the shape of two facing arrows (Fig. 1a) ${ }^{10,19}$. A Co film was then electrodeposited to close the gap between the electrodes using a cobalt sulphate electroplating bath under constant voltage conditions. Figure $1 \mathrm{~b}$ shows a typical image of the formation of a contact area between the electrodes. The impedance between the two patterned electrodes was monitored in situ, under low a.c. excitation $(<4 \mathrm{mV}$ r.m.s. $)$, at a frequency of $211 \mathrm{~Hz}$. A $1-\mathrm{k} \Omega$ resistor, in series with the sample, was used to limit the current to a few microamps r.m.s. and as shunt for measuring the current flow. The phase of the lock-in detection unambiguously confirms the resistive nature of the contacts, in strong contrast to the noisy capacitive component found when the impedance of the system is larger than approximately $100 \mathrm{k} \Omega$, a value at which the electrochemical bath contributes more than a few percent to the measured impedance. 
The entire electrochemical cell was rotated by a step motor in a magnetic field of $1 \mathrm{~T}$ produced by an electromagnet. Both the angle and the conductance were synchronically recorded as a function of time. The angular rotation was performed from $0^{\circ}$ to $180^{\circ}$, with $0^{\circ}$ corresponding to the magnetic field in the electrode plane and $90^{\circ}$ to the field perpendicular to the plane (Fig. 2a). The operational bandwidth limits the time interval between two measurements to about $10 \mathrm{~ms}$. To increase the probability of accomplishing a scan of full angular range during a quantum conductance plateau, we selected a $T=20 \mathrm{~s}$ rotation period, which provides an angular resolution of about $0.2^{\circ}$.

Of significant concern is the possibility of creating conductance changes as a result of mechanical displacements of the electrodes ${ }^{18,19,26}$. Our experimental set up, in which the electrodes are bonded to the substrate, minimizes mechanical effects ${ }^{19}$. The magnetostrictive change of the size of the nanocontact can be estimated by multiplying the length of unbound part of the contact by the saturation magnetostriction coefficient, resulting in a value smaller than $0.1 \AA$ for Co. This relatively small quantity is of little concern for the angular dependence studied here, because the polycrystallinity of our samples makes magnetostriction independent of the sample angle at the saturation magnetic field. A control experiment was carried out by performing rotation prior to applying the magnetic field during the first $100 \mathrm{~s}$ to verify that the conductance does not change with angle (first part of the trace in Fig. 2b). Possible substrate deformations were also ruled out by performing experiments using $\mathrm{Au}$ and Ni substrates, which have significantly different elastic properties, but provide identical BAMR properties. Finally, similar experiments performed on Ni nanocontacts revealed anisotropic magnetoresistance deviating from bulk AMR, but not showing clear quantized changes, therefore ruling out the occurrence of steps due to mechanical artefacts (see Supplementary Information, Fig. S4), as has also been found by others ${ }^{21}$. A possible explanation for the absence of quantized conductance in $\mathrm{Ni}$ contacts is the weakened magnetism of $\mathrm{Ni}$ in constrained geometries ${ }^{28}$.

\section{Theory}

Band structure calculations were performed using a simple tightbinding model. We consider a monoatomic wire and assume that only $d$ states of one spin band are present at the Fermi energy. Note that we are interested only in the conductance change due to the rotation of the magnetization with respect to the axis of the wire. The contribution to the conductance from other bands crossing the Fermi energy such as $s$ bands gives only an additive correction to the overall conductance, which is not affected by the magnetization orientation. We assume that the exchange splitting is large compared with the spin-orbit interaction so that we can ignore the influence of the spin mixing on the band structure. Thus, the band structure represents the multiplet of $d$ states corresponding to the total electronic momentum quantum number $L=2$. It is split by the crystal field of axial symmetry into two doublets, $\{|+1\rangle \pm|-1\rangle\} / \sqrt{ } 2$ and $\{|+2\rangle \pm \mid-$ $2\rangle\} / \sqrt{2}$, and a singlet $|0\rangle$. The singlet belongs to the $A_{1}$ symmetry. The doublets have $E_{1}$ and $E_{2}$ symmetry and are further split by the spin-orbit interaction $E_{\mathrm{SO}}=\lambda \mathbf{L S}$, where $\mathbf{L}$ is the orbital momentum, $\mathbf{S}$ is the spin momentum, and $\lambda$ is a constant of the spin-orbit interaction. The splitting of the $E$ symmetry bands changes from zero for the magnetization perpendicular to the wire axis (Fig. 4c) to a maximum for the magnetization parallel to the wire axis (Fig. 4a). Ab initio calculations reveal that the magnitude of the splitting is of the order of $10^{-1} \mathrm{eV}$ (ref. 13; see this reference for more complete symmetry arguments).

\section{Author contributions}

A.S. and C.Z. performed the experiment and analysed the data. E.Y.T. performed the theory, J.R. provided guidance for the electrochemistry part, and B.D. conceived and designed the experiment.

\section{Acknowledgements}

This work was supported by the Nebraska Research Initiative and the National Science Foundation through the Materials Research Science and Engineering Center (grant DMR-0213808) and the Chemistry Division (grant CHE-0518644). B.D. acknowledges support from a 'Chaire d'excellence' fellowship of the French ANR.

\section{Competing interests statement:}

The authors declare no competing financial interests.

\section{References}

1. Sharvin, Y. V. A possible method for studying Fermi surfaces. Sov. Phys. JETP 21, 655-656 (1965).

2. Montie, E. A. et al. Observation of the optical analogue of quantized conductance of a point contact. Nature 350, 594-595 (1991).

3. Krans, J. M. et al. The signature of conductance quantization in metallic point contacts. Nature 375, 767-769 (1995).

4. Ohnishi, H., Kondo, Y. \& Takayanagi, K. Quantized conductance through individual rows of suspended gold atoms. Nature 395, 780-785 (1998).

5. Oshima, H. \& Miyano, K. Spin-dependent conductance quantization in nickel point contacts. Appl. Phys. Lett. 73, 2203-2205 (1998).

6. Ono, T., Ooka, Y. \& Miyajima, H. $2 \mathrm{e}^{2} / \mathrm{h}$ to $\mathrm{e}^{2} / \mathrm{h}$ switching of quantum conductance associated with a change in nanoscale ferromagnetic domain structure. Appl. Phys. Lett. 75, 1622-1624 (1999).

7. Imamura, H., Kobayashi, N., Takahashi, S. \& Maekawa, S. Conductance quantization and magnetoresistance in magnetic point contacts. Phys. Rev. Lett. 84, 1003-1005 (2000)

8. Versluijs, J. J., Bari, M. A. \& Coey, J. M. D. Magnetoresistance of half-metallic oxide nanocontacts. Phys. Rev. Lett. 87, 026601 (2001).

9. Chung, H. et al. Universal scaling of ballistic magnetoresistance in magnetic nanocontacts. Phys. Rev. Lett. 89, 287203 (2002).

10. Yang, C.-S., Zhang, C., Redepenning, J. \& Doudin, B. In-situ magnetoresistance of Ni nanocontact. Appl. Phys. Lett. 84, 2865-2867 (2004).

11. Viret, M. et al. Magnetoresistance through a single nickel atom. Phys. Rev. B 66, 220401 (2002).

12. Chopra, H. D., Sullivan, M. R., Armstrong, J. N. \& Hua, S. Z. The quantum spin-valve in cobalt atomic point contacts. Nature Mater. 4, 832-837 (2005).

13. Velev, J., Sabirianov, R., Jaswal, S. S. \& Tsymbal, E. Y. Ballistic anisotropic magnetoresistance. Phys. Rev. Lett. 94, 127203 (2005).

14. Wolf, S. A. et al. Spintronics: a spin-based electronics vision for the future. Science 294, 1488-1495 (2001).

15. Johnson, M. Magnetoelectronics (Elsevier B.V., Amsterdam, 2004).

16. Ney, A., Pampuch, C., Koch, R. \& Ploog, K. H. Programmable computing with a single magnetoresistive element. Nature 425, 485-487 (2003).

17. Sahoo, S. et al. Electric field control of spin transport. Nature Phys. 1, 99-102 (2005).

18. Brumfiel, G. Magnetic effect sends physicists into a spin. Nature 426, 110 $110(2003)$

19. Yang, C.-S., Zhang, C., Redepenning, J. \& Doudin, B. Anisotropy magnetoresistance of quantum ballistic nickel nanocontacts. J. Magn. Magn. Mater. 286, 186-188 (2005).

20. Viret, M. et al. Giant anisotropic magneto-resistance in ferromagnetic atomic contacts. Eur. Phys. J. B 51, 1-4 (2006).

21. Bolotin, K. I., Kuemmeth, F. \& Ralph, D. C. Anisotropic magnetoresistance and anisotropic tunneling magnetoresistance due to quantum interference in ferromagnetic metal break junctions. Phys. Rev. Lett. 97, 127202 (2006).

22. Keane, Z. K., Yu, L. H. \& Natelson, D. Magnetoresistance of atomic-scale electromigrated nickel nanocontacts. Appl. Phys. Lett. 88, 062514 (2006).

23. Thomson, W. On the electrodynamic qualities of metals. Proc. Royal Soc. 8 , 546-550 (1857).

24. McGuire, T. R. \& Potter, R. I. Anisotropic magnetoresistance in ferromagnetic 3d alloys. IEEE Trans. Magn. 11, 1018-1038 (1975).

25. Landauer, R. Spatial variation of currents and fields due to localized scatterers in metallic conduction. IBM J. Res. Dev. 1, 223-231 (1957).

26. Egelhoff, W. F. Jr et al. Artifacts in ballistic magnetoresistance measurements. J. Appl. Phys. 95, 7554-7559 (2004).

27. Sabirianov, R., Solanki, A. K., Burton, J. D., Jaswal, S. S. \& Tsymbal, E. Y. Domain wall magnetoresistance in Co nanowires. Phys. Rev. B 72, 054443 (2005).

28. Burton, J. D., Sabirianiov, R. F., Jaswal, S. S., Tsymbal, E. Y. \& Mryasov, O. N. Magnetic moment softening and domain wall resistance in Ni nanowires. Phys. Rev. Lett. 97, 077204 (2006). 


\section{Supplementary Information}

\section{Quantized Magnetoresistance in Atomic-Size Contacts}

Andrei Sokolov, Chunjuan Zhang, Evgeny Y. Tsymbal, Jody Redepenning, and Bernard Doudin

Complementary experimental data are presented, illustrating several statements made in the paper.

Fig.S1 shows nanocontact impedance measured in-situ during growth and dissolution of nanocontact. Test experiments were performed initially on $\mathrm{Ni}$ to check the reproducibility of deposition/dissolution experiments (without applying any magnetic field). Successive reduction (-0.55 V vs Ag/AgCl reference electrode) and oxidation (0.0 V vs $\mathrm{Ag} / \mathrm{AgCl})$ of $\mathrm{Ni}$ ions in a solution of $\mathrm{Ni}$ sulfamate result in slow plating and dissolution of the ferromagnetic metal (Fig.S1-a). Simultaneous measurement of the phase of the AC current flowing between the two sides of the nanocontact (Fig.S1-b) provides a clear signature of the occurrence of a resistive contact, in contrast to the capacitive noisy contribution of the electrolytic bath. Spikes in the measured voltage reveal transients related to a sudden change in electrochemical conditions. Figs.S1-c,d display the respective conductance versus time curves corresponding to plating (Figs.S1-c) and dissolution (Figs.S1-d) of the nanocontact.
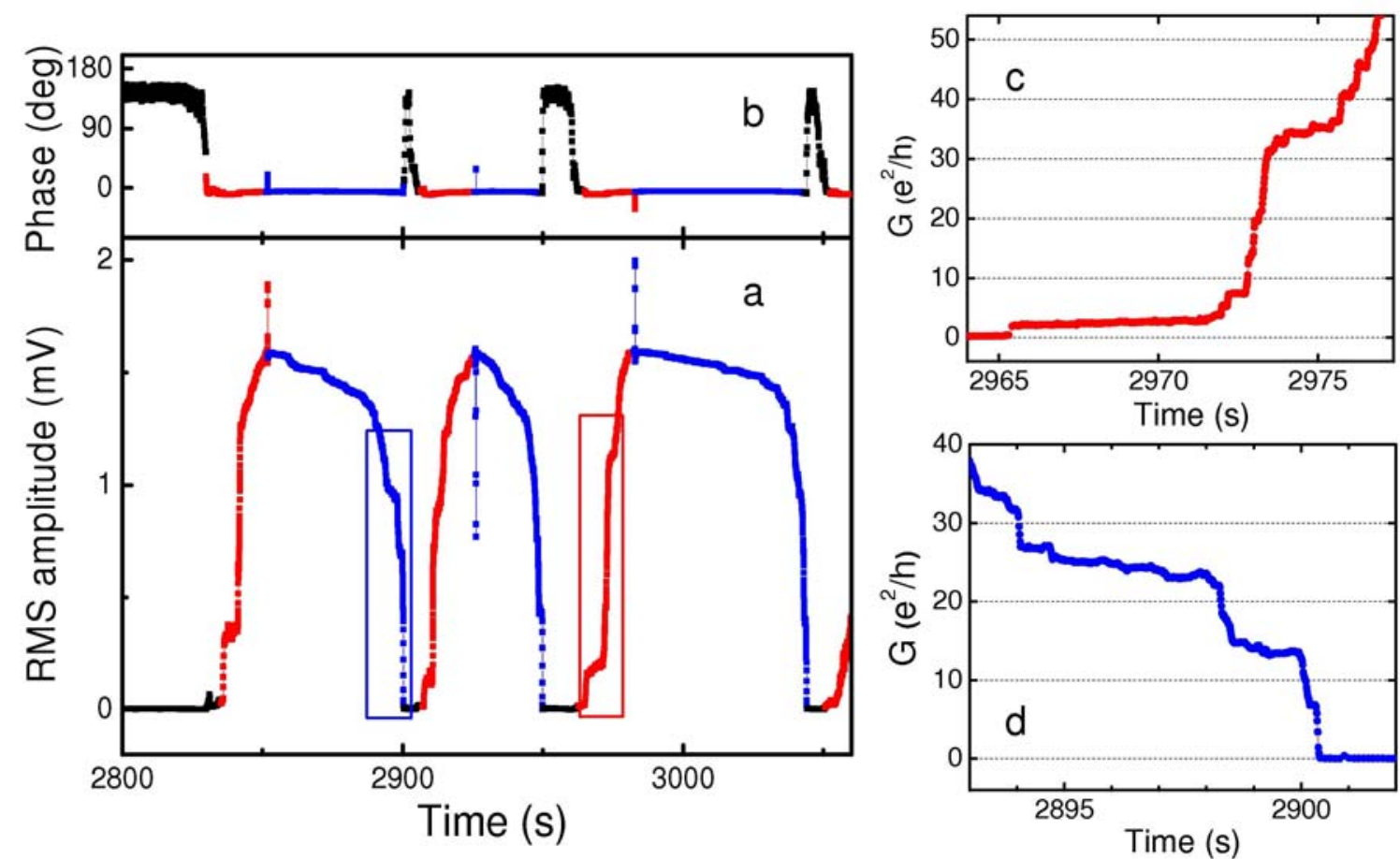

Fig.S1 In-situ nanocontact impedance during growth and dissolution. Time trace of amplitude (a) and phase (b) of the voltage drop through a $1 \mathrm{k} \Omega$ shunt resistor in series with the impedance between the two electrodes indicate three regimes. Black dots correspond to impedance values larger than $100 \mathrm{k} \Omega$, related to a noisy non-zero phase, and the absence of direct electrical contact between electrodes. Red dots correspond to reduction of $\mathrm{Ni}$ ions, and slow contact closure. Blue dots correspond to a slow dissolution of the contact and conductance decreasing with time. c, d: Conductance versus time curves evaluated from the data of panel (a) for two areas indicated by rectangles of the corresponding colour. 
Fig.S2 shows that BAMR is insensitive to the magnitude of the applied magnetic field when the field exceeds the saturation field of $8 \mathrm{kOe}$. This allows us to rule out possible artefacts in the measurements related to effects dependent on the magnitude of the applied magnetic field.

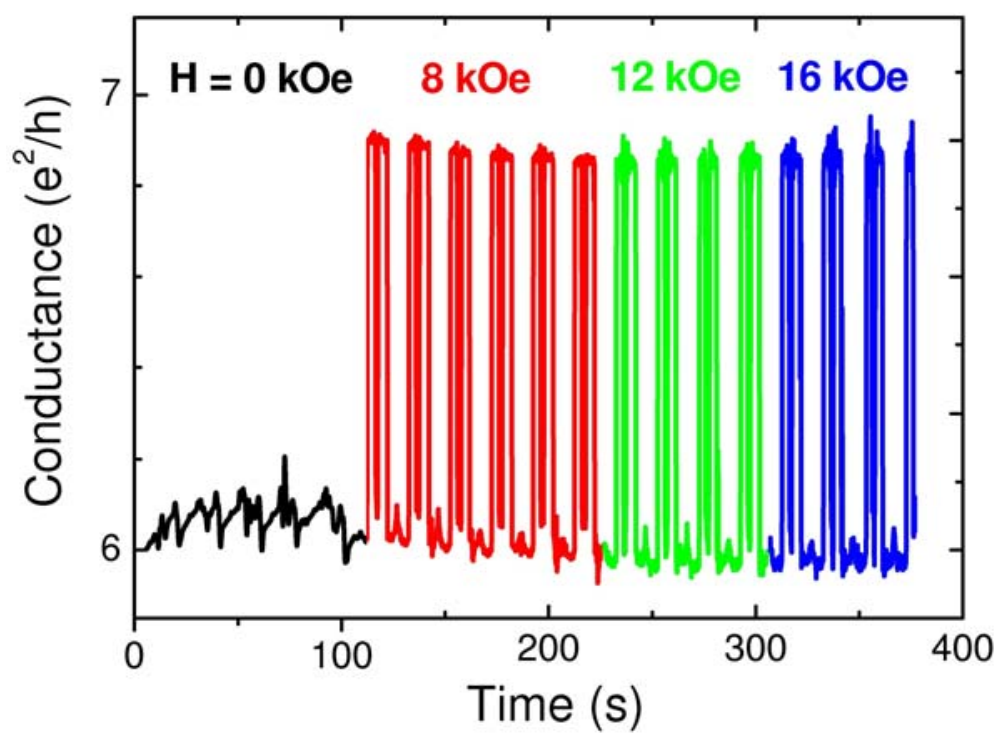

Fig.S2 BAMR with varying applied saturating field values. Time trace of the conductance of a Co nanocontact, during the rotation of the sample in applied magnetic fields of several different magnitudes. The rotation period corresponds to the time periodicity of the oscillating conductance. These data show that no significant influence on the BAMR is observed when the applied field is higher than the saturation field.

Fig.S3 shows the typical distribution of the magnetization angles at which the conductance changes abruptly, illustrating the discussion on statistics of this switching due to thermal effects given in the main paper.

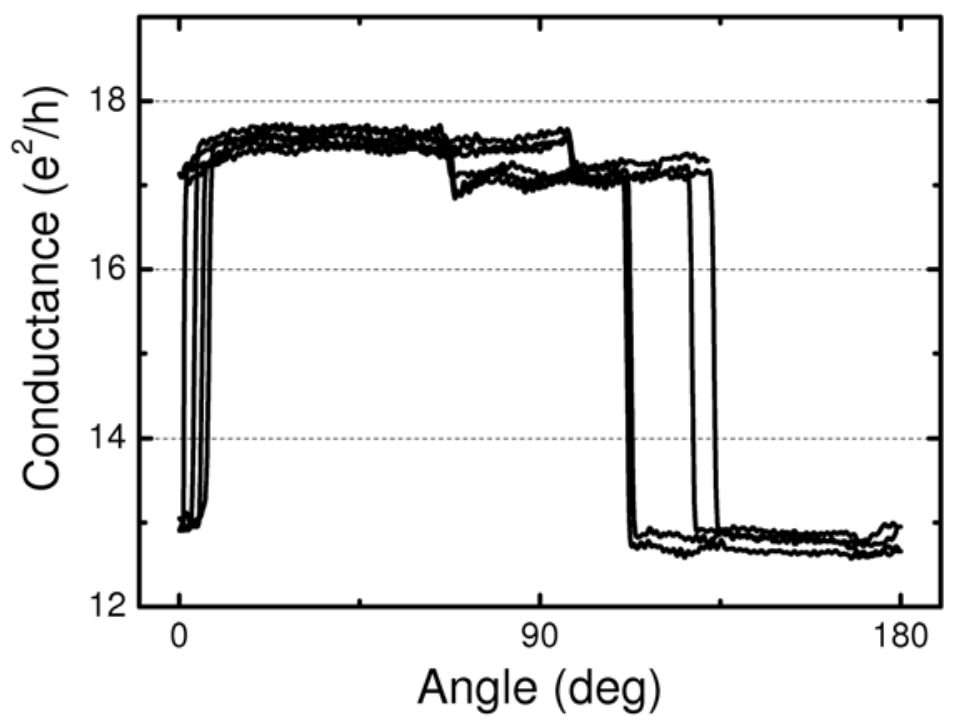


Fig.S3 Superposition of several BAMR traces. Angular variation of the conductance of a Co contact, for which four sweeps occurred within the time stabilization of a conductance step.

Fig.S4 demonstrates results of AMR measurements on Ni nanocontacts, not showing quantized angular variation. The curves do not follow a simple $\cos ^{2} \Theta$ law but display systematically maximum and minimum conductance at $\Theta=90^{\circ}$ and $\Theta=0$ respectively, similarly to what is expected for macroscopic diffusive samples.

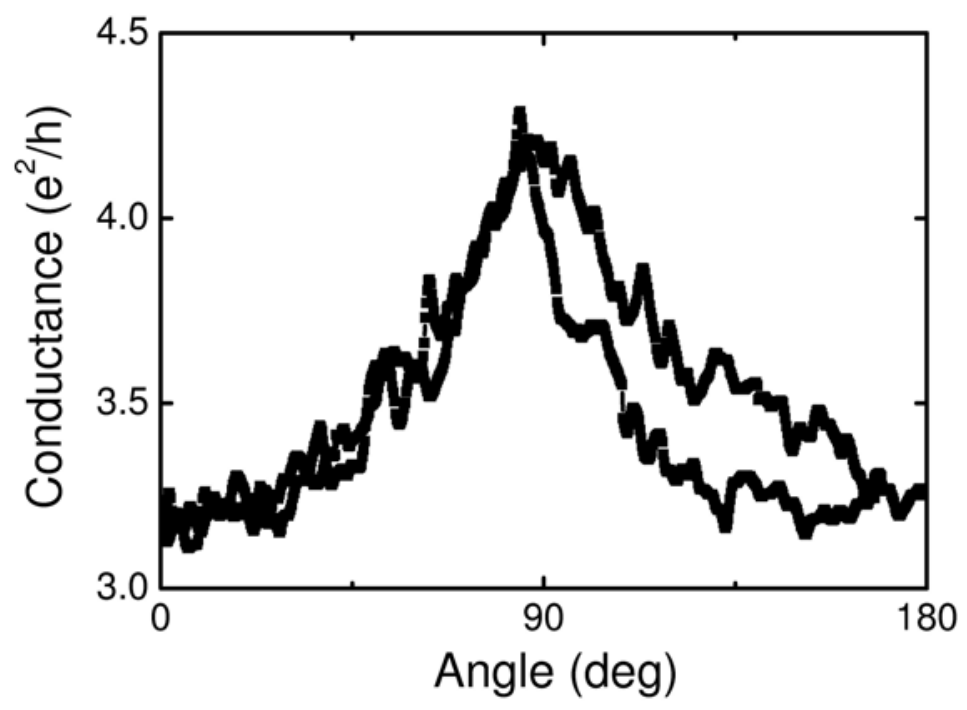

Fig. S4 AMR of Ni nanocontact. Angular variation of the conductance of a Ni contact, for which no clear steps are observed, and maximum (minimum) of the conductance systematically observed at $\Theta=90^{\circ}$ $\left(\Theta=0^{\circ}\right)$. 